\title{
Aplicación del criterio de mínimo costo en la determinación del óptimo tamaño de planta para una empresa cafetera en Antioquia
}

\section{Application of the minimum cost criteria for the determination of the optimal plant size in a coffee company in Antioquia}

\author{
Daniela Morales-Cespedes a \& Eduardo Duque-Grisales ${ }^{b}$ \\ ${ }^{a}$ Administradora Financiera. Grupo de Investigación en Dirección de Empresas. Institución Universitaria Esumer. \\ danielamc-14@hotmail.com \\ ${ }^{b}$ Magister en Ingeniería. Grupo de Investigación en Dirección de Empresas. Institución Universitaria Esumer. \\ Eduardo.duque@esumer.edu.co
}

Recibido: noviembre 9, 2019. Aceptado: diciembre 22, 2019.

\begin{abstract}
Resumen
Este trabajo propone la utilización del criterio de costo mínimo para la determinar el óptimo tamaño de planta en la empresa "Café de Mañana". Con esta aplicación, se busca la identificación del modelo de producción que minimice los costos de inversión, tendiendo en cuenta que se debe satisfacer la futura demanda de dos líneas de preparación de café empaquetado, tostado y molido. Así, se puede establecer una medida de la competitividad para los pequeños productores que buscan incrementar sus réditos dentro de un sistema desequilibrado donde la oportunidad de competir a nivel de precios es escasa, frente a las escalas que manejan productores más grandes. Para las empresas resulta imprescindible tener totalmente interiorizado este concepto, pues tener un criterio para determinar un tamaño de planta óptimo será muy importante para ser más eficiente.
\end{abstract}

Palabras Clave: función de producción, función de costos, economía de escala, costo mínimo, Costo marginal, empresa cafetera.

\begin{abstract}
This paper proposes the use of the minimum cost criterion to determine the optimum plant size in the "Café de Mañana" company. This application looks for the identification of the production model that minimizes investment costs, considering that the future demand for two lines of preparation of packaged, roasted, and ground coffee must be met. Thus, a measure of competitiveness can be established for small producers seeking to increase their revenues within an unbalanced system where the opportunity to compete at the price level is low compared to the scales managed by larger producers. For companies, it is essential to have this concept fully internalized, since having a criterion to determine an optimal plant size will be very important to increase organizational efficiency.
\end{abstract}

Keywords: production function, cost function, economy of scale, minimal cost, marginal cost, coffee company.

\section{INTRODUCCIÓN}

El café es uno de los productos de mayor consumo en todo el mundo. La Organización Internacional del Café (ICO) presenta este grano como uno de los productos primarios más valiosos y más importante para la economía mundial [1]. Colombia ha sido tradicionalmente uno de los principales productores de café y sus cosechas se caracterizan por la gran suavidad y calidad que tienen sus granos, gracias a los diferentes pisos térmicos que se hallan dentro del territorio nacional [2]. Sin embargo, la comercialización se da en términos del grano de café verde sin procesar, sin el valor agregado como el obtenido con el proceso de tostado y molido requerido para su venta final.

Algunas empresas cafeteras han querido implementar procesos de secado y molido en el café verde que producen, pero han tenido obstáculos de infraestructura y de segmentación del mercado que no han posibilitado el pleno desarrollo de esta actividad [3], especialmente dificultades con el tamaño óptimo de su planta de producción. La capacidad de producir de una empresa, que es un indicador de su tamaño, no debe provenir del azar, debe ser el resultado de una decisión cuidadosamente analizada. Esto significa que cuando existen dos o más posibilidades para lograr un mismo objetivo deberán desarrollarse los estudios de las diferentes alternativas sometiendo la decisión final a una rigurosa evaluación [4]. En el caso del tamaño de la planta para un cierto

\section{Citar como:}

Daniela Morales-Cespedes \& Eduardo Duque-Grisales. "Aplicación del criterio de mínimo costo en la determinación del óptimo tamaño de planta para una empresa cafetera en Antioquia" Revista CINTEX, Vol. 24(2), pp. 55-63. 2019. 
Aplicación del criterio de mínimo costo en la determinación del óptimo tamaño de planta para una empresa cafetera en

Application of the minimum cost criteria for the determination of the optimal plant size in a coffee company in Antioquia

volumen de producción, la capacidad podría estar sustentada en factores fijos en el corto plazo; sin embargo, estos factores suelen variar todos en el mediano o en el largo plazo, habida cuenta de su disponibilidad, particularmente de algunos factores que determinan las diferentes capacidades de producción tales como locales, plantas y equipos pesados [5].

En este artículo se pretende determinar el tamaño óptimo de la empresa "Café de Mañana". La finca de donde se extrae la materia prima y la planta donde se localiza la producción se encuentra en la vereda Miraflores, ubicada en el municipio de Venecia, a 60 kilómetros de Medellín, Antioquia. Esta empresa tiene un efecto muy positivo social y ambientalmente en el sector, pues impulsa el desarrollo cafetero de la mano de los caficultores cercanos, mejorando la infraestructura de la vereda y la productividad en los procesos, a esto se le suma que la zona es muy rica en recursos naturales de flora y fauna. La empresa busca la comercialización de café procesado en cuatro presentaciones, y para ello es indispensable dimensionar el tamaño de planta óptimo con el que pueda operar la empresa, teniendo en cuenta que los costos fijos que sustentan su capacidad de producción y la estructura de la planta pueden considerarse como variables de decisión a largo plazo.

En principio, se toma en cuenta que existe una relación funcional entre el monto de la inversión y el tamaño de la planta, lo cual permite considerar la inversión (I) como una variable dependiente de la capacidad de producción de la planta (Q). Por lo tanto, la escala óptima de inversión es aquella que minimizará los costos pero atenderá la demanda futura [6]. En consecuencia, la definición del tamaño de planta está asociada a la determinación de costos de inversión y los costos operativos que se derivan del proceso productivo [7]. De esta manera, se tiene en cuenta que en la definición del tamaño de planta están implícitas ciertas variables como la estructura del mercado, las alternativas tecnológicas, la localización de la planta, el precio de los insumos y la capacidad financiera de la firma.

El resto del artículo está organizado de la siguiente manera. En el capítulo dos se presenta una revisión de literatura sobre el tamaño de planta óptimo. En el capítulo tres se presenta la metodología utilizada en este artículo para definir el procedimiento adecuado para obtener el tamaño de planta óptimo de la empresa "Café de mañana". Posteriormente, se presentan el análisis de resultados y las conclusiones.

\section{REVISIÓN DE LITERATURA}

\subsection{LAS FUNCIONES DE COSTO MEDIO DE CORTO Y LARGO PLAZO}

El tamaño óptimo de la planta puede ser considerada una variable dinámica como resultado de sucesivas expansiones impulsadas por el crecimiento de una demanda variable en el tiempo [8]. En este caso, el análisis del horizonte temporal es de trascendental importancia debido a que los costos fijos correspondientes al tamaño de la planta de corto plazo pueden volverse variables en función de las expansiones necesarias para la satisfacción de una demanda futura. Por lo tanto, será necesario encontrar un tamaño de planta de costo mínimo (óptimo) sobre las funciones de costo medio de corto y de largo plazo.

\subsubsection{COSTO MEDIO DE CORTO PLAZO (CMCP)}

Una vez la firma se compromete con una planta de un tamaño particular, sólo puede variar su volumen de producción modificando otros factores, diferentes al tamaño de la planta, como por ejemplo las cantidades de insumos, o contratando otro turno de trabajadores o aumentando la disponibilidad de materias primas. Esto quiere decir que, en el corto plazo, la firma no puede ajustar el tamaño de su planta de producción, el cual está asociado a un costo fijo, pero sí puede variar sus volúmenes de producción modificando los niveles de los factores variables tales como mano de obra y materia prima. Es decir, para cada tamaño de planta, existe una función de Costo Medio de Corto Plazo, denotada por CMCP, que incluye los costos anuales de todos los insumos relevantes tales como trabajo directo, materiales y energía, así como los costos fijos anualizados de la misma planta, incluido el costo de oportunidad. La figura 1 ilustra el caso de una firma cuya producción puede realizarse en una planta con tres posibles tamaños, a saber: pequeña, CMCP (Q); mediana, CMMPL $(Q)$ y grande, CMLPL (Q). Además, en negrilla, se ilustra la envolvente que representa la Función de Costo Medio de Largo Plazo, como se detalla más adelante.

En esta gráfica, puede apreciarse que, si la firma conociera previamente la cantidad que planea producir, para minimizar sus costos, debería escoger el tamaño de planta que arroje el menor Costo Medio de Corto Plazo, CMCP, para el nivel deseado de producción. Por ejemplo, tal como se ilustra en la figura 1, el tamaño óptimo de la planta será pequeño para un nivel de producción Q1; mediano, para un volumen de producción Q2; grande, para una producción de Q3. Es decir, a mayor nivel de producción, mayor deberá ser el tamaño de la planta. Además, cuando el nivel de producción es Q1, la reducción en el costo medio que resulta de seleccionar una planta pequeña, en vez de una grande, suele ser significativo y puede calcularse como la diferencia de sus costos medios, así: el Costo Medio de Corto Plazo en la Planta Grande asociado a la 
producción Q1 menos el Costo Medio de Corto Plazo en la Planta Pequeña de la producción Q1, esto es: CMCPG (Q1) - CMCP (Q1). Esta reducción en el Costo Medio de Corto Plazo no solo puede presentarse por los ahorros en los costos fijos de la planta, sino, además, porque a este tamaño de planta la firma puede ajustar más eficientemente el resto de sus operaciones. Por ejemplo, cuando una empresa produce un volumen Q1 en la planta grande, podría utilizar más trabajo para controlar el flujo interno de materiales, mientras que la planta pequeña podría permitir flujos más ágiles y ahorros de trabajo [10].

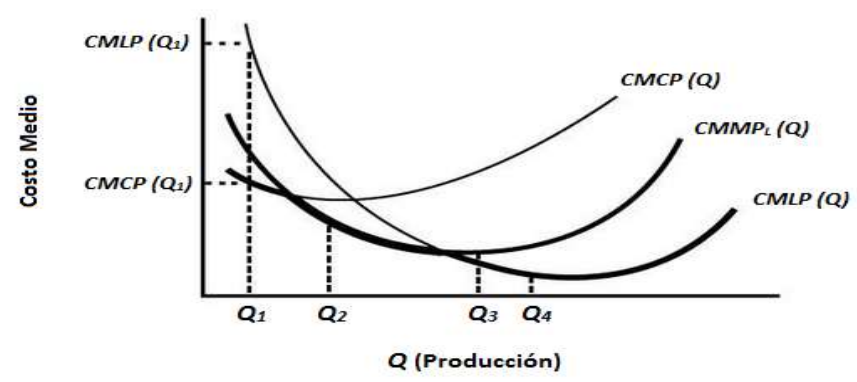

Figura 1. Funciones de Costo Medio de Corto y de Largo Plazo.

\subsubsection{Costo Medio de LaRgo Plazo (CMLP)}

La función de Costo Medio de Largo Plazo, CMLP, es la envolvente de las funciones de Costo Medio de Corto Plazo, representada por la línea en negrita en la figura 1, la cual muestra el menor costo medio que puede alcanzarse en un nivel particular de producción, si la firma pudiera ajustar su tamaño de planta de manera continua, es decir, si hubiera tecnología disponible para cualquier punto de la curva de Costo Medio de Largo Plazo. Esta es la función de costo medio que la firma enfrenta antes de comprometerse con un tamaño de planta particular. Sin embargo, en la práctica, la demanda es más dinámica que la capacidad y en consecuencia los ajustes del tamaño de la planta sobre una curva de este tipo obligan a considerar excesos de capacidad para responder al mercado estratégicamente, es decir, para maximizar beneficios por medio de posiciones dominantes que podrían derivarse de posiciones monopólicas.

\subsection{LOS COSTOS HUNDIDOS}

Los costos hundidos (costo de montaje de la planta) actúan creando una ventaja competitiva de la firma ya establecida en un mercado sobre otra firma que desea entrar al mercado [11]. Esto se debe a que, generalmente, la inversión asociada a la planta es irreversible y, en tal caso, estos costos no pueden recuperarse. Sin embargo, la capacidad de producción de la planta establecida puede actuar como una barrera de entrada para nuevas firmas y permitir a la primera disfrutar de cierto poder monopólico, al que le es más difícil aspirar a las nuevas empresas que no tienen este costo hundido.

No obstante, para una firma instalada se presenta el riesgo de que surja una nueva tecnología que la desplace, lo cual sería posible sólo si los ahorros en los costos operativos de la nueva tecnología superaran la diferencia entre los costos invertidos en la planta instalada y los costos fijos de la nueva planta de la empresa que va a entrar. Esta posición competitiva de la empresa instalada se produce porque puede ofrecer al mercado un precio igual a su costo marginal, el cual es menor que el costo medio de una empresa entrante, la que solo podría entrar con un precio por lo menos igual al costo medio de la firma ya instalada.

\subsection{EL EXCESO DE CAPACIDAD}

Marvin B. Lieberman (1987), explica el concepto tradicional de exceso de capacidad considerando las razones que se describen a continuación [12]:

- En algunas industrias, solamente resulta económico agregar capacidad mediante grandes incrementos de producción, esto es, incrementando la capacidad en forma discreta para grandes saltos de producción discontinuos. Sin embargo, para hacer frente a incrementos futuros en la demanda, estas industrias pueden experimentar períodos en los cuales las empresas muestran exceso de capacidad. Por lo tanto, será necesario buscar un punto óptimo en el que debe hacerse la expansión de capacidad y su magnitud para no soportar costos ociosos durante largos períodos de tiempo. 
Aplicación del criterio de mínimo costo en la determinación del óptimo tamaño de planta para una empresa cafetera en

Application of the minimum cost criteria for the determination of the optimal plant size in a coffee company in Antioquia

- La reducción en la actividad económica general o la caída en la demanda de una empresa pueden generar exceso de capacidad. En el caso de una demanda con comportamiento cíclico, podrán presentarse unos periodos con exceso de capacidad y otros con déficit.

- Las firmas que participan en ciertos tipos de industrias pueden ser rentables solo operando a plena capacidad. La entrada de una firma nueva que busca quedarse con parte de los beneficios podría crear excesos de oferta, es decir, de capacidad.

No obstante, según el mismo autor, las firmas además pueden utilizar el exceso de capacidad por motivos estratégicos. Este sería el caso cuando una empresa establecida puede mantener dicho exceso de capacidad para evitar la entrada de eventuales competidores que desearían disputarle el mercado. La razón es que cuando se tiene exceso de capacidad, puede incrementarse la producción a un costo relativamente bajo. En caso de competencia imperfecta, la empresa establecida podría considerar conveniente incrementar significativamente sus niveles de producción, aprovechando sus economías de escala para bajar precio y, desde luego, incrementar sus ventas. El efecto de esto, intencionado o no, será una reducción importante en los beneficios de la empresa que intenta ingresar al mercado, posterior a su entrada. Si estos beneficios son menores que los costos hundidos de la entrada (o sea los costos fijos), la firma entrante se mantendrá por fuera del mercado. El monopolista establecido podría incluso decidir no utilizar toda su capacidad y usar su recurso ocioso como una amenaza creíble de que incrementará su producción en caso de que se produzca la entrada.

De esta manera, como se enuncia a continuación, Lieberman (1987) especifica las condiciones bajo las cuales, mediante el exceso de capacidad, una empresa establecida puede prevenir de manera exitosa la entrada de nuevos competidores.

- La empresa establecida debe tener una ventaja en costos, que puede aprovechar en caso de que se produzca la entrada y una eventual guerra de precios.

- El crecimiento de la demanda debe ser lento. De lo contrario, la demanda podría sobrepasar rápidamente la capacidad y habría mercado para todos los entrantes.

- La inversión hecha para generar exceso de capacidad deberá ser considerada como un costo hundido antes de que se produzca una nueva entrada. De otra manera, la empresa entrante podría forzar a la firma establecida a dar marcha atrás en caso de que se desatara una guerra de precios. No hay que olvidar que cuando se toman los costos para batallar en guerra de precios, el tomador de la decisión debe ignorar los costos hundidos y considerar sólo los costos evitables (costos marginales).

La bondad del exceso de capacidad para competir en precios se ha ilustrado en una vieja clase de microeconomía donde se explica cómo los ritmos de crecimiento de los costos medios, en situación de costos variables pequeños, permiten usar el exceso de capacidad para disputar clientes a otros productores [13]. Para procesos de producción con costos fijos altos, el costo marginal (variable) puede ser pequeño y en consecuencia es posible bajar los costos medios unitarios para un amplio rango de producción. Solamente cuando la producción se acerque a su capacidad, los costos medios empiezan a elevarse drásticamente por efecto de la congestión, lo cual impide seguir aprovechando las economías de escala. Esto significa que las propiedades físicas del proceso de producción, cuando se satura, exigen a las empresas expandir la capacidad con un aumento de los costos fijos, lo cual hace que el costo marginal comience a hacer subir significativamente el costo medio. Esta situación de cercanía al nivel de congestión da una señal al planificador de la producción para ir decidiendo la próxima expansión.

A largo plazo, en una competencia como ésta, donde la demanda no se recupera, lo que puede pasar es que las empresas subsistan basadas en las economías de escala. Si la capacidad de la firma es la específica de la industria (es decir, solamente puede ser usada para producir en esta industria), las firmas no tendrán otra opción más que permanecer allí hasta que la planta alcance el final de su vida útil o hasta que se recupere la demanda. Si ésta no se recupera, las empresas pueden aguantar un periodo prolongado de exceso de capacidad con precios por debajo del costo medio.

\subsection{LAS ECONOMÍAS dE ESCALA Y LA FUNCIÓN DE COSTOS}

Como generalmente sucede, la tecnología a utilizar permite la ampliación de la capacidad productiva solo por tramos. En tal sentido, para la optimización del tamaño de la planta deberá evaluarse fundamentalmente la relación costo-volumen [14] por efecto de las economías y des-economías de escala, lo cual permite mostrar el impacto de la incorporación de factores (costos de inversión) en el nivel de producción. Se dice que existen economías de escala si al multiplicar todos los factores por una constante $t$, el nivel de producción será mayor que $t$ veces la producción inicial, lo cual puede ilustrarse diciendo que si, por ejemplo, se duplican los factores, 
la producción se incrementará a más del doble. Estas economías pueden ser internas, inherentes al proceso productivo, o externas, propias del proceso de contratación con los proveedores de factores de producción. En consecuencia, cuando el proceso productivo exhibe economías de escala, los costos unitarios disminuyen al aumentar los niveles o volúmenes de producción. Así entonces, la escala o magnitud de los costos, C, depende de decisiones relacionadas con la magnitud de la ampliación del sistema existente, o sea, con el tamaño de una nueva instalación. Dichas decisiones son importantes porque el tamaño de la ampliación de la planta afectará la estructura de costos.

En síntesis, las economías de escala se crean cuando los costos de un sistema no aumentan con igual rapidez que su tamaño [15]. En los estudios del diseño de la planta y de selección de los equipos y la maquinaria necesarios para elaborar la producción planeada, deberán considerarse las condiciones bajo las cuales va a operar dicha tecnología y si tendrán impacto en el rendimiento.

Sin embargo, si los costos unitarios de un proceso productivo disminuyen a medida que este aumenta su capacidad, esto no significa que los tamaños para su ampliación deban concebirse a la mayor escala posible para así obtener los costos unitarios más bajos. Lo que significa es que el analista de planta deberá considerar las expansiones frente a los costos, ya que una gran ampliación de capacidad pude incrementar los costos económicos por dos razones: primero, porque los recursos empleados para ampliar el sistema permanecerían improductivos hasta que se necesite la capacidad adicional; segundo, estos recursos no podrían aprovecharse en usos mucho más productivos en otras esferas de la economía, lo cual representa el costo de oportunidad de la ampliación. Por lo tanto, la solución óptima podría encontrarse al lograr un equilibrio entre las posibles economías de escala y los costos de oportunidad menores asociados a las inversiones de menor escala [16].

Para ilustrar esta relación entre una función de costos y las economías de escala, conviene examinar la función de costos que se representa mediante la ecuación (2) donde $\mathbf{K}$ es el tamaño de la inversión asociada a la adición de capacidad de la instalación; $\boldsymbol{\alpha}$ es el factor de escala; $\mathbf{Q}$ es la capacidad o tamaño de la planta, que puede medirse en miles de metros cúbicos por día o en litros por segundo, o en otra unidad de capacidad como tránsito promedio diario si se tratara de una vía; A es un coeficiente cuyo valor depende de las unidades de medida tanto de los costos como de la capacidad.

$$
K=A * Q^{a} \quad \text { Ecuación (1) }
$$

donde $\alpha$ es un índice de la medida en que se alteran los costos según se modifica la capacidad (Elasticidad). Esto es, ante una expansión, la variación de los costos con respecto a la variación de la capacidad será mayor, igual o menor que 1. Si $\alpha>1$, significa que ante un pequeño porcentaje de variación de la capacidad los costos reaccionan cambiando más allá de ese porcentaje; si $\alpha=1$, significa que ante variaciones en la capacidad los costos varían en la misma proporción, y si $\alpha<1$, significa que el porcentaje de variación en la capacidad es mayor al porcentaje de variación en los costos, es decir esta variación es inelástica a los costos; lo cual podría denotarse como se muestra a continuación.

$$
\boldsymbol{a}=\frac{\Delta \boldsymbol{K}}{\Delta \boldsymbol{Q}} * \frac{\boldsymbol{Q}}{\boldsymbol{K}} \quad \text { Ecuación (2) }
$$

El valor de $\alpha$ para esta clase de equipos de secado y molido se averigua en el mercado y equivale $\alpha=0.9$.

\section{CASO DE ESTUDIO: EMPRESA "CAFÉ DE MAÑANA"}

"Café de Mañana" se diferencia por ser un café de origen, lo que significa que al ser un café de una sola finca lo hace más atractivo y valioso sin mencionar que sus características en cuanto a cuerpo, aroma, acidez y sabor le han merecido puntajes entre 85 y 90 calificándolo como un café excelente de origen especial. La empresa comercializará libras de café tostado y molido en diferentes cantidades:

$$
\begin{aligned}
& >\mathrm{P}_{1} \text { : Café tostado de } 2.500 \mathrm{~g} \\
& >\mathrm{P}_{2} \text { : Café tostado de } 500 \mathrm{~g} \\
& >\mathrm{P}_{3} \text { : Café molido de } 2.500 \mathrm{~g} \\
& >\mathrm{P}_{4} \text { : Café molido de } 500 \mathrm{~g}
\end{aligned}
$$

Para el cálculo del tamaño de la planta se tendrán en cuenta las siguientes hipótesis:

- El período de vida útil de la planta será, por lo menos, de 20 años ( $T=20$ años). 
Aplicación del criterio de mínimo costo en la determinación del óptimo tamaño de planta para una empresa cafetera en

Application of the minimum cost criteria for the determination of the optimal plant size in a coffee company in Antioquia

- Los equipos adquiridos tendrán una depreciación de 10 años.

- El costo de oportunidad del inversionista a largo plazo es del $6 \%$ anual

- La producción y las ventas de los dos productos al momento de arrancar la planta y de salir al mercado son:

$>M_{01}: 21.000$ unidades

$>M_{02}: 63.000$ unidades

$>$ Mo3: 16.000 unidades

$>$ Mo4: 33.000 unidades

- La capacidad de la planta para I producción de este tipo de productos se calcula en megavatios y está dado por:

$>\mathrm{b}_{1}: 0.1052$ unidad/MWh

$>\quad b_{2}: 0.3125$ unidad/MWh

$>\mathrm{b}_{3}: 0.1818$ unidad/MWh

$>\mathrm{b}_{4}: 0.2000$ unidad/MWh

- Los precios de venta netos, después de gastos variables y de descuentos y costos proporcionales a ellos (que denotamos $p_{i}$ ), y los costos de sus insumos, comisiones de ventas, energía eléctrica y otros variables, por unidad de cada producto (que denotamos por $q_{i}$ ) son los siguientes:

$>\mathrm{p}_{1}: 5$ USD/unidad

$>\mathrm{p}_{2}: 22$ USD/unidad

$>p_{3}: 6$ USD/unidad $q_{1}: 2$ USD/unidad $\mathrm{q}_{2}: 10.5$ USD/unidad

$>\mathrm{p}_{4}: 28$ USD/unidad $\mathrm{q}_{3}: 3.5$ USD/unidad q4: 18 USD/unidad

\subsection{PROYECCIÓN DE LA DEMANDA}

Los estudios previos de la demanda permiten pronosticar que la demanda de los cuatro productos va a crecer de manera geométrica, siguiendo las funciones
$M_{n}(t)=M_{0 n} e^{k t}$
Ecuación (3)

donde $k$ es la tasa de crecimiento, equivalente al $5 \%$.

La planta trabajará holgadamente, con capacidad sobrante, hasta el momento $t=\boldsymbol{\tau}$ cuando ocurra que las cuatro producciones copen su capacidad, lo que será cuando

$$
\sum_{i=1}^{4} \frac{M_{i}(\tau)}{b i}=Q
$$

Las utilidades por unidad de tiempo, antes de impuestos, pueden escribirse:

$$
\begin{array}{lcc}
U_{1}(t)=\Sigma M_{1}(t) \cdot v_{i}-F-d\left(A Q^{\alpha}+K_{2}\right) & \text { Para } 0<t<\tau \quad \text { Ecuación (5) } \\
U_{2}(t)=\Sigma P_{i}(t) \cdot v_{i}-F-d\left(A Q^{\alpha}+K_{2}\right) & \text { Para } 0<t<T e=10 a \text { ños } \quad \text { Ecuación (6) } \\
U_{3}(t)=\Sigma M_{3}(t) \cdot v_{1}-F-d\left(A Q^{\alpha}+K_{2}\right) & \text { Ecuación }(7)
\end{array}
$$

en donde $v_{i}=P_{i}-q_{i}$ y equivale al valor agregado por producto y $\mathrm{K}_{2}$ corresponde al valor de los activos depreciables que no dependen del tamaño $Q$ del proyecto.

Por lo tanto, el valor presente de las utilidades de los 20 años, después de impuestos, es: 
$Y(Q, t)=\left[\int_{0}^{t} U_{1}(t) \cdot e^{-r t} \cdot d t+\int_{t}^{T e} U_{2}(t) \cdot e^{-r t} \cdot d t+\int_{T e}^{T} U_{3}(t) \cdot e^{-r t} \cdot d t\right](1-X)$ Ec. (8)

Entonces el valor agregado por año y por megavatio de capacidad de uso, cuando la planta se cope, y siga trabajando copada es:

$$
v_{e=} f_{1} \cdot v_{1} \cdot b_{1}+f_{2} \cdot v_{2} \cdot b_{2}+f_{2} \cdot v_{2} \cdot b_{2}+f_{3} \cdot v_{3} \cdot b_{3}+f_{4} \cdot v_{4} \cdot b_{4} \quad \text { Ecuación (9) }
$$

La duración como vida útil que se prevé para la planta es de $T=20$ años. Usaremos en este problema como criterio de optimización del proyecto para calcular su tamaño óptimo, el de hacer máximo el valor descontando de las utilidades de los 20 años, en valor presente, después de impuestos. Esto requiere, como bien se sabe, las dos condiciones:

$$
\begin{array}{ll}
\boldsymbol{d} \boldsymbol{Y}(\boldsymbol{Q}, \boldsymbol{t}) \boldsymbol{d} \boldsymbol{Q}=\mathbf{0} & \text { Ecuación (10) } \\
d^{2} \boldsymbol{Y}(\boldsymbol{Q}, \boldsymbol{t}) \boldsymbol{d} \boldsymbol{Q}^{\mathbf{2}}=\mathbf{0} & \text { Ecuación (11) }
\end{array}
$$

Cuando la planta está copada (en $\mathrm{t}=\boldsymbol{\tau}$ y después de $\tau$ ) se tendrá para la producción $P_{i}$ de cada producto, que:

$$
P_{i}(t) / b_{i}=\mathcal{Q} \quad \text { Ecuación (12) }
$$

Y la parte "o cuota" de cada producto en el "product-mix" será:

$$
f_{1}=P_{i}(t) /\left(b_{i} \mathcal{Q}\right) \quad \text { Ecuación }(13)
$$

De manera que:

$$
P_{i=\mathcal{Q}} b_{i} f_{1} \quad \text { Ecuación (14) }
$$

La utilidad contable de los 20 años futuros, descontada a su valor presente en $t=0$, y después de impuestos, vale:

$$
\begin{aligned}
& \frac{d Y(Q)}{d Q}=(1-x)\left[\left(D_{0}+D_{1}+\tau+D_{2} \cdot \tau\right)\right] e^{-r}\left(\frac{d t}{d Q}\right)+\int_{t}^{T} V e^{*} \cdot e^{-r \tau} \cdot d \tau-V e^{*} \cdot Q \cdot e^{-r \tau}\left(\frac{d \tau}{d Q}\right)-A \cdot \alpha \cdot \frac{Q^{\alpha-1}\left(1-e^{-r t}\right)}{r T e}+ \\
& \left(\frac{V e^{*}}{r}\right)\left(e^{-r \tau}-e^{-r T}\right) \quad \text { Ecuación (15) }
\end{aligned}
$$

Y si despejamos a $\mathcal{Q}$ se obtiene;

$$
\mathcal{Q}=\left[\frac{\alpha A}{T e V e^{*}} \frac{1-e^{-r T e}}{e^{-r \tau}-e^{-r T}}\right]^{1 /(1-\alpha)} \quad \text { Ecuación (16) }
$$

Para verificar la segunda condición del óptimo, calculamos la segunda derivada de $Y(Q, t)$ respecto a $Y$, para obtener:

$$
\frac{d^{2} Y(Q)}{d^{2}}=\frac{a(1-a) A}{T e Q^{2-a}} \frac{1-e^{-r T}}{r}-V^{*} e^{-r t}\left(\frac{d \tau}{d Q}\right) \quad \text { Ecuación (17) }
$$

De acuerdo con la expresión que ya obtuvimos para la segunda derivada de $\mathrm{Y}$ respecto a $\mathcal{Q}$, confirmando que sea negativo para que efectivamente sea el óptimo:

$$
\frac{d^{2} Y(\mathcal{Q})}{d Q^{2}}=\frac{a(1-a) A}{T e Q^{2-a}} \frac{1-e^{-r T e}}{r}-V^{*} e^{-r \tau} \frac{d \tau}{d Q} \quad \text { Ecuación (18) }
$$

Resolviendo el problema por medio de métodos numéricos 'de newton y con ayuda del software matemático Matlab, se obtienen los siguientes resultados (ver Tabla 1). 
Aplicación del criterio de mínimo costo en la determinación del óptimo tamaño de planta para una empresa cafetera en

Application of the minimum cost criteria for the determination of the optimal plant size in a coffee company in Antioquia

Tabla 1. Resultados de la simulación

\begin{tabular}{|l|l|}
\hline Variable & Valor \\
\hline Tiempo óptimo $\tau$ & 12.84 años \\
\hline Capacidad óptima $Q$ & $27.94 \mathrm{MW}$ \\
\hline Valor agregado año $\boldsymbol{V} \boldsymbol{e}^{*}$ & 87.6 USD/MWh \\
\hline $\begin{array}{c}\text { Utilidad después de } \\
\text { impuestos Y }\end{array}$ & 90.400 USD \\
\hline
\end{tabular}

\section{ConClusiones Y TRABAJO FUtURO}

La decisión sobre el tamaño de la planta de producción no debe obedecer a situaciones coyunturales de corto plazo, sino que deberá optimizarse frente al dinamismo de la demanda. Ello implica que debe ser mayor el tamaño que la demanda actual, pero esta capacidad debe adaptarse frente a las expectativas de su crecimiento. Por lo tanto, el análisis de los rangos de variación del tamaño permitirá definir los límites dentro de los cuales se fijará la capacidad de producción de la firma. Estos rangos de variación del tamaño no solo indican que la capacidad óptima de un proceso productivo se forma por medio de ampliaciones, sino también que es importante fijar un calendario de expansiones que estará vinculado al tipo de proceso productivo y a las características de flexibilidad de la tecnología.

Así entonces, desde un enfoque de este tipo, el valor actual de los costos de una planta se calculará sumando los costos descontados desde el año en que se inicia su montaje, año cero, hasta el último año en que haya de realizarse el ajuste de planta. Por lo tanto, para la empresa "Café de Mañana" es necesario una planta de producción comercial de $30 \mathrm{MW}$ que se ajusta a las necesidades de proyección de crecimiento en el tiempo y que le dejará unas utilidades de 90.400 USD acorde a la proyección de la demanda.

\section{REFERENCIAS}

[1] L. C. OLMOS, E. A. DUQUE, y E. RODRIGUEZ, «State of the art of coffee drying technologies in Colombia and their global development», Rev. Espac., vol. 38, n. ${ }^{\circ}$ 29, 2017.

[2] O. L. Ocampo-López y L. M. Alvarez-Herrera, «Tendencia de la producción y el consumo del café en Colombia», Apunt. CENES, vol. 36, n. ${ }^{\circ}$ 64, pp. 139-165, 2017.

[3] H. Araque Salazar, «Variables tecnológicas que determinan la productividad de las fincas cafeteras del departamento de Caldas», 2015.

[4] A. Infante Villareal, Evaluacion financiera de proyectos de inversion. Bogota; San Juan, [P.R.]: Norma, 1984.

[5] E. Zapa Pérez, «Impacto de la Gestión por Procesos en la Innovación de las Organizaciones», Rev. CINTEX, vol. 19, pp. 23-37, 2014.

[6] N. Sapag Chain y R. Sapag Chain, Preparación y evaluación de proyectos. Santafé de Bogotá: McGrawHill, 1995.

[7] F. A. González, J. J. Gómez, y D. F. Amaya, «Multispectral image processing in coffee and cocoa crops», Rev. CINTEX, vol. 22, n. ${ }^{\circ}$ 2, pp. 51-67, dic. 2017, doi: 10.33131/24222208.294.

[8] T. Daugaard, L. A. Mutti, M. M. Wright, R. C. Brown, y P. Componation, «Learning rates and their impacts on the optimal capacities and production costs of biorefineries», Biofuels Bioprod. Biorefining, vol. 9, n. ${ }^{\circ} 1$, pp. 82-94, 2015.

[9] D. Besanko, D. Dranove, M. Shanley, y S. Schaefer, Economics of strategy. John Wiley \& Sons, 2009.

[10] J. Echavarría, «Condiciones Laborales y Productivas en Microempresas de Confección de la ciudad de Medellín», Rev. CINTEX, vol. 20, n. 2, pp. 79-95, 2015.

[11] M. J. Roberts y J. R. Tybout, «La decisión de exportar en Colombia: un modelo empírico de entrada con costos hundidos», Rev. Ens. Sobre Política Económica Vol 16 No 31 Junio 1997 Pág 61-100, 1997.

[12] M. B. Lieberman, «Excess capacity as a barrier to entry: An empirical appraisal», J. Ind. Econ., pp. 607-627, 1987.

[13] R. S. Pindyck, D. L. Rubinfeld, E. Rabasco, y L. Toharia, Microeconomía. Hertfordshire (United Kingdom): Prentice Hall, 1995. 
[14] O. L. García Serna, Administración financiera: fundamentos y aplicaciones. Cali: Prensa Moderna Impresores, 1999.

[15] J. Atack, Estimation of economies of scale in nineteenth century United States manufacturing. Routledge, 2018.

[16] S. Ruiz, J. Patiño, A. Marquez-Ruiz, J. Espinosa, E. Duque, y P. Ortiz, «Optimal Design of a Diesel-PVWind-Battery-Hydro Pumped POWER system with the Integration of ELECTRIC vehicles in a Colombian Community», Energies, vol. 12, n. ${ }^{\circ} 23$, p. 4542, nov. 2019, doi: 10.3390/en12234542. 